\title{
HEAT TRANSFER IN DARCY-FORCHHEIMER FLOW OF TANGENT HYPERBOLIC FLUID OVER AN INCLINED PLATE WITH JOULE HEATING
}

\author{
Darapuneni Purna Chandar Rao ${ }^{1,2}$, Swaminathan Thiagarajan ${ }^{2}$ \\ Vajha Srinivasa Kumar ${ }^{3}$ \\ ${ }^{I}$ Research Scholar, Department of Mathematics, JNTUH College of Engineering, JNTU Kukatpally \\ Hyderabad - 500085, Telangana, India \\ ${ }^{2}$ Department of Mathematics, Matrusri Engineering College, Saidabad, Hyderabad - 500059 \\ Telangana, India \\ ${ }^{3}$ Department of Mathematics, JNTUH College of Engineering, JNTU Kukatpally, Hyderabad- \\ 500085, Telangana, India \\ dprao@matrusri.edu.in,drst_rajan@yahoo.co.in,vajhasrinu@gmail.com
}

Received: 31 March 2021; Accepted: 12 August 2021

\begin{abstract}
Tangent hyperbolic fluid is one of the non-Newtonian fluids in which the constitutive equation is valid for low and high shear rates and used mostly in laboratory experiments and industries. The Darcy-Forchheimer flow model is substantial in the fields where the high flow rate effect is the common phenomenon, for instance, in petroleum engineering. With these things in mind, in this article, we analysed the mixed convective dissipative Darcy-Forchheimer flow of tangent hyperbolic fluid by an inclined plate with Joule heating. Flow administering equations were altered as nonlinear ODEs and then resolved using shooting strategy. Pertinent outcomes are explained through graphs. It is discovered that fluid velocity minifies with the rise in the power law index parameter and Forchheimer number. It is detected that the thermal buoyancy parameter minimizes fluid temperature, and the magnetic field parameter ameliorates the same. What's more, we noticed that Forchheimer number minimizes the skin friction coefficient, and the heat transfer rate is minified with the larger Eckert number. Furthermore, we have verified our results with former results for the Nusselt number and noticed a satisfactory agreement.
\end{abstract}

MSC 2010: 76W05, 76E06, 76A05, 80A32

Keywords: inclined plate, tangent hyperbolic fluid, Darcy-Forchheimer, Joule heating, shooting strategy, Eckert number

\section{Introduction}

Joule warming depicts the interaction where the energy of an electric flow is transformed into heat as it courses through an obstruction. Joule heating is utilized in the industrial process and in multiple devices, for example, in Laboratory water 
bath (laboratory equipment utilized for reactions at warm temperature). Khader [1] considered Joule heating and quiescent (Newtonian) fluid flow through an exponentially stretching sheet. He noticed that the Prandtl number meliorates the Nusselt number. Patel and Singh [2] and Mittal et al. [3] investigated micropolar fluid flow by a stretching surface with Joule heating and found that the temperature is minified by a larger magnetic parameter. Shamshuddin et al. [4] elucidated the squeezing flow of MHD fluid among riga plates with Joule heating. They observed the escalation in the temperature parameter with a larger solutal Biot number. Waqas et al. [5] emphasized that the Schmidt number is useful to ameliorate both transfer rates (heat, mass) in their scrutiny on thixotropic fluid flow with Joule heating. Kumar et al. [6] carried out an examination to elaborate the characteristics of entropy generation in Williamson (nano) fluid flow by a stretching sheet and remarked that the radiation parameter meliorates fluid temperature. Devi and Sharma [7] discovered that the temperature minifies with the larger mixed convection parameter in the scrutiny of MHD fluid flow with variable permeability through a cone. Alaidrous and Eid [8] considered Joule heating and higher order chemical reaction and studied three dimensional hyperbolic tangent fluid flow by an extending surface immersed in a porous medium. They detected that the porosity parameter decelerates the fluid velocity. Investigation of Carreau fluid flow by a slendering elongated sheet with Joule heating is done by Gayatri et al. [9] and acknowledged that the chemical reaction parameter slenderizes the fluid concentration. Shoaib et al. [10] implemented Lobatto IIIA methodology via MATLAB to inspect the dissipative hybrid nanofluid flow by a rotating disk with Joule heating. They observed that the porosity parameter minimizes both radial and azimuthal friction factor.

A fluid in which viscosity is not constant and depends on shear rate is termed as non-Newtonian fluid. Non-Newtonian fluid flows are useful in several areas including mining, plastic processing and chemical processing industries. Tangent hyperbolic fluid is one of the non-Newtonian fluids in which the constitutive equation is valid for low and high shear rates and used mostly in laboratory experiments and industries. Examples of tangent hyperbolic fluids are blood, paint and ketchup. Khan et al. [11] scrutinized the Casson fluid flow by a stretched surface with homogeneous-heterogeneous reactions and observed that the homogeneous reaction heat parameter ameliorates the fluid temperature. Nayak et al. [12] and Khan and Alzahrani [13] considered stretching surface and discussed the entropy generation on the dissipative flow of various non-Newtonian fluids with thermal radiation. They found that the transverse and axial wall shear stress ameliorates with the larger Weissenberg number. Later, various researchers [14-18] considered activation energy and analysed different non-Newtonian fluid flows over distinct geometries.

The Darcy-Forchheimer flow model is substantial in the fields where a high flow rate effect is the common phenomenon, for instance, in petroleum engineering. Ibrahim and Khan [19] used the finite difference method to investigate the Darcy flow of CNT mixed nanofluid flow with viscous dissipation. They noticed 
that the porosity parameter minimizes the fluid velocity. Khan and Alzahrani [20] elucidated the Darcy-Forchheimer flow of hybrid nanofluid among two stretchable revolving disks with second order velocity slip and remarked that the Forchheimer number minimizes the axial velocity. Recently, Khan [21] and Khan and Alzahrani $[22,23]$ considered the Darcy-Forchheimer model and scrutinized various nanofluid flows with viscous dissipation and thermal radiation.

After careful observation of the aforementioned literature, we noticed that no study is reported on the Darcy-Forchheimer flow of tangent hyperbolic fluid by an inclined plate. To fill this gap, we have examined a MHD mixed convective flow of tangent hyperbolic fluid through an inclined plate with Joule heating. The Runge-Kutta fourth order based shooting technique is applied to resolve the transmuted equations. Outcomes are exhibited via graphs for two instances i.e., suction and injection.

\section{Formulation}

In this work, we took mixed convective tangent hyperbolic fluid flow via an inclined plate with Joule heating and viscous dissipation. The Darcy-Forchheimer model is used in the momentum equation. We presume that the plate is inclined with an angle $\gamma$ and elongated with velocity $u_{w}=c x$. The flow pattern is displayed in Figure 1. Temperatures of the surface and ambient are denoted by $T_{w}$ and $T_{\infty}$. Additionally, we neglected the induced magnetic field.

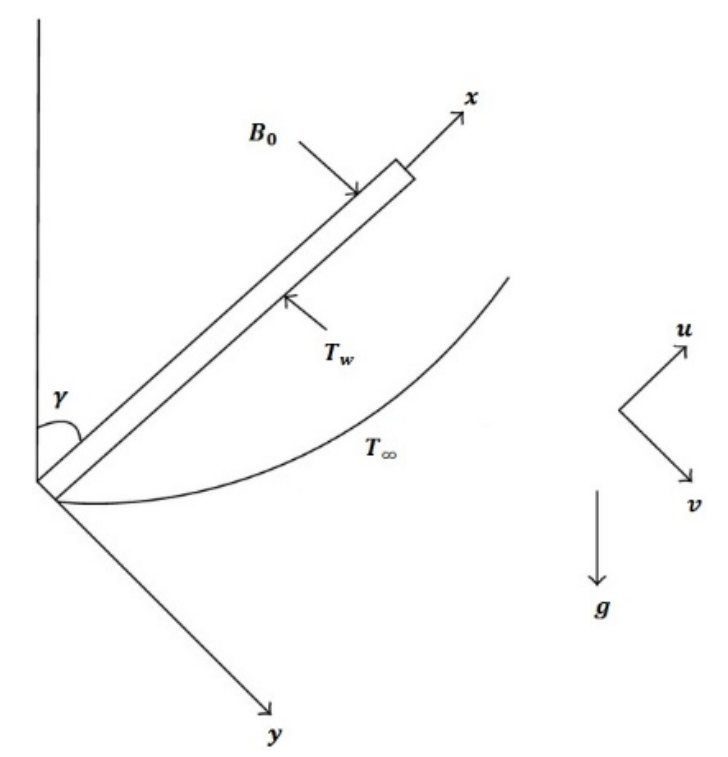

Fig. 1. Flow form of tangent hyperbolic fluid by an inclined plate 
Equations describing the flow are [24, 25]

$$
\begin{gathered}
\frac{\partial v}{\partial y}+\frac{\partial u}{\partial x}=0 \\
u \frac{\partial u}{\partial x}+v \frac{\partial u}{\partial y}=v(1-m) \frac{\partial^{2} u}{\partial y^{2}}+\sqrt{2} v m \Gamma\left(\frac{\partial u}{\partial y}\right) \frac{\partial^{2} u}{\partial y^{2}}+g \beta_{T} \cos \gamma\left(T-T_{\infty}\right)-u \frac{v}{\mathrm{~K}}-F u^{2}-\frac{\sigma B_{0}^{2}}{\rho} u \\
u \frac{\partial T}{\partial x}+v \frac{\partial T}{\partial y}=\frac{k}{\rho C_{p}} \frac{\partial^{2} T}{\partial y^{2}}+\frac{v}{C_{p}}\left(\frac{\partial u}{\partial y}\right)^{2}+\frac{1}{\rho C_{p}} Q^{*}\left(T-T_{\infty}\right)+\frac{\sigma B_{0}^{2}}{\rho C_{p}} u^{2}
\end{gathered}
$$

with the conditions

$$
\left.\begin{array}{l}
u=u_{w}, v=-v_{w}, T=T_{w} \text { at } y=0 \\
u \rightarrow 0, T \rightarrow T_{\infty} \text { as } y \rightarrow \infty
\end{array}\right\}
$$

where: $u, v$ are velocity constituents in two directions $(x, y), \rho$ - fluid density, $v$ - kinematic viscosity, $\sigma$ - electrical conductivity, $\beta_{T}$ - thermal expansion volumetric coefficient, $g$ - gravity acceleration, $K$ - permeability of porous medium, $F\left(=\frac{C_{b}}{x \sqrt{K}}\right)-$ inertia coefficient, $B_{0}-$ magnetic field intensity, $C_{b}-$ drag coefficient, $\Gamma$ - time dependent material constant, $m$ - power law index parameter, $T$ - fluid temperature, $k$ - thermal conductivity, $C_{p}$ - specific heat capacity, $Q^{*}$ - (dimensional) heat source parameter, $\mu$-dynamic viscosity, $v_{w}$ - permeability of the porous surface.

Using the similarity transformations

$$
\eta=y \sqrt{\frac{u_{w}}{v x}}, u=u_{w} f^{\prime}(\eta), v=-\sqrt{c v} f(\eta), \theta(\eta)=\frac{T-T_{\infty}}{T_{w}-T_{\infty}},
$$

continuity equation (1) is satisfied and equations (2)-(3) are changed as:

$$
\begin{gathered}
{\left[(1-m)+m W e f^{\prime \prime}\right] f^{\prime \prime}+f f^{\prime \prime}+\delta \cos \gamma \theta-(\Lambda+M) f^{\prime}-(1+F r) f^{\prime 2}=0} \\
\frac{1}{\operatorname{Pr}} \theta^{\prime \prime}+f \theta^{\prime}+M E_{c} f^{\prime 2}+E_{c} f^{\prime \prime 2}+H \theta=0
\end{gathered}
$$

and conditions in (4) are altered as

$$
\left.\begin{array}{l}
f(0)=S, f^{\prime}(0)=1, \theta(0)=1 \text { i.e., at } \eta=0 \\
f^{\prime}(\infty) \rightarrow 0, \theta(\infty) \rightarrow 0 \text { i.e., as } \eta \rightarrow \infty
\end{array}\right\}
$$


The Weissenberg number We, local Reynold's number $\mathrm{Re}_{x}$, local thermal Grashoff number $G r$, mixed convection parameter $\delta$, Eckert number $E_{c}$, Prandtl number $\operatorname{Pr}$, porosity parameter $\lambda$, heat source parameter $H$, Forchheimer number $F r$, and suction/injection coefficient $S$ are indicated as:

$$
\left.\begin{array}{l}
W e=\frac{\sqrt{2} \Gamma c^{3 / 2} x}{\sqrt{v}}, \operatorname{Re}_{x}=\frac{x u_{w}}{v}, G r=\frac{g \beta_{T}\left(T_{w}-T_{\infty}\right) x^{3}}{v^{2}}, \delta=\frac{G r}{\operatorname{Re}_{x}^{2}}, E_{c}=\frac{u_{w}^{2}}{C_{p}\left(T_{w}-T_{\infty}\right)}, \\
\operatorname{Pr}=\frac{\mu C_{p}}{k}, \lambda=\frac{v}{K c}, H=\frac{Q^{*}}{c\left(\rho C_{p}\right)}, F r=\frac{C_{b}}{\sqrt{K}}, S=\frac{v_{w}}{\sqrt{c v}}
\end{array}\right\} .
$$

The Nusselt number $N u_{x}$, surface friction drag $C f_{x}$ are defined as:

$$
N u_{x}=\left.\frac{x q_{w}}{k\left(T_{w}-T_{\infty}\right)}\right|_{y=0}, C f_{x}=\left.\frac{\tau_{w}}{\frac{1}{2} \rho u_{w}{ }^{2}}\right|_{y=0}
$$

where: $q_{w}=-k \frac{\partial T}{\partial y}, \tau_{w}=\left[(1-m) \frac{\partial u}{\partial y}+\frac{m \Gamma}{\sqrt{2}}\left(\frac{\partial u}{\partial y}\right)^{2}\right]$.

By using (5), we can write (9) in a non-dimensional form as

$$
\left(\operatorname{Re}_{x}\right)^{-1 / 2} N u_{x}=-\theta^{\prime}(0),\left(\operatorname{Re}_{x}\right)^{1 / 2} C f_{x}=2\left[(1-m) f^{\prime \prime}(0)+\frac{m}{2} W e\left(f^{\prime \prime}(0)\right)^{2}\right] .
$$

\section{Results and discussion}

We used the Runge-Kutta $4^{\text {th }}$ order based shooting technique to solve transmuted equations (6)-(7) with the conditions (8). Results are exhibited via graphs in two instances i.e., suction and injection. Figure 2 exhibits that the power lax index parameter reduces the thickness of the hydrodynamic layer. Therefore, it lowers the velocity profile. Generally, the larger Forchheimer number produces the drag force, which causes the fluid flow to slow down. That means, resistive force is enhanced for the fluid with the rise in the Forchheimer number. Therefore, the rise in the Forchheimer number diminishes the fluid velocity (Fig. 3). When we raise the thermal buoyancy parameter, more buoyancy forces rise in the fluid. So, escalation in fluid velocity occurs with the larger thermal buoyancy parameter (Fig. 4). Figure 5 depict that the Weissenberg number lowers fluid velocity (fluid thickness increases with the larger Weissenberg number). Typically, the Weissenberg number is a ratio between the shear rate time and the relaxation time. Hence, for the larger $W e$, the fluid becomes thicker, and consequently, the fluid velocity decreases. 


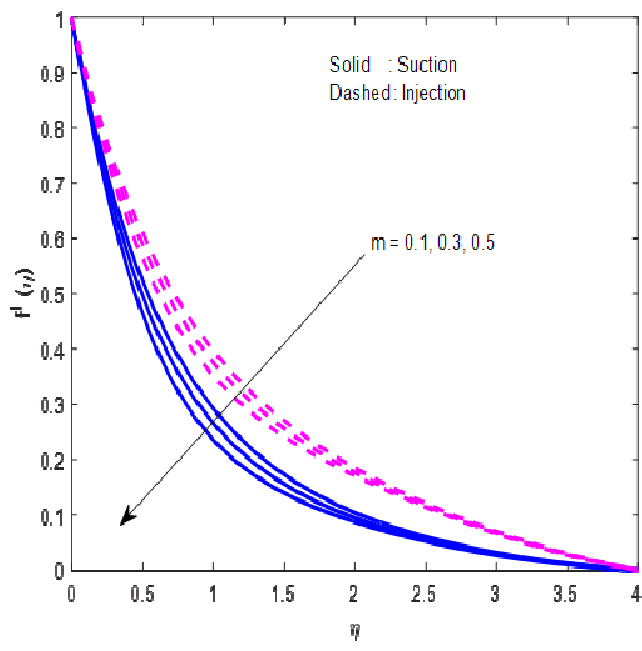

Fig. 2. Consequence of $m$ on velocity profile

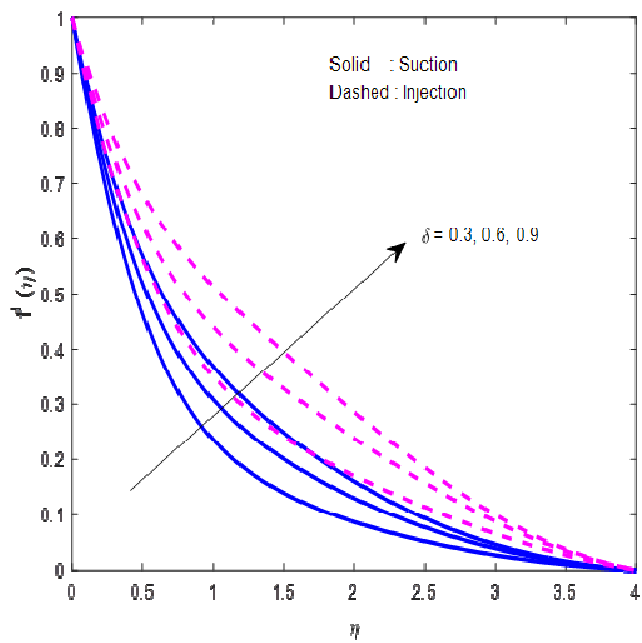

Fig. 4. Consequence of $\delta$ on velocity profile

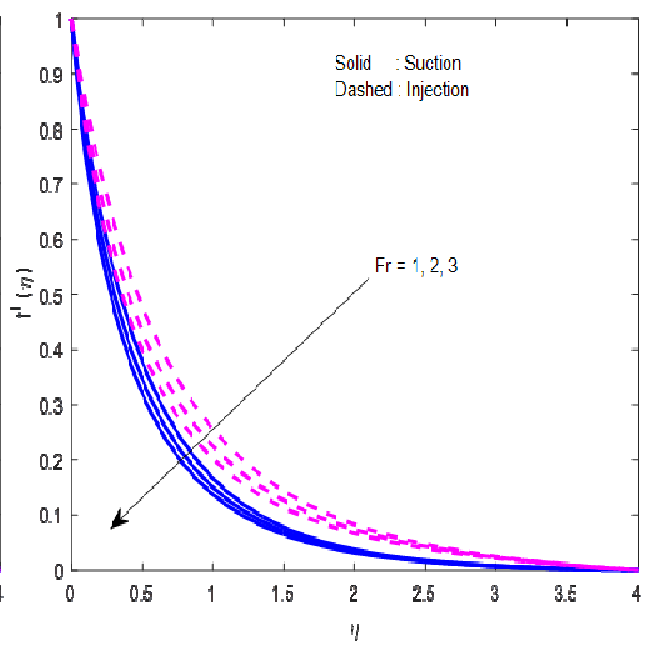

Fig. 3. Consequence of $\mathrm{Fr}$ on velocity profile

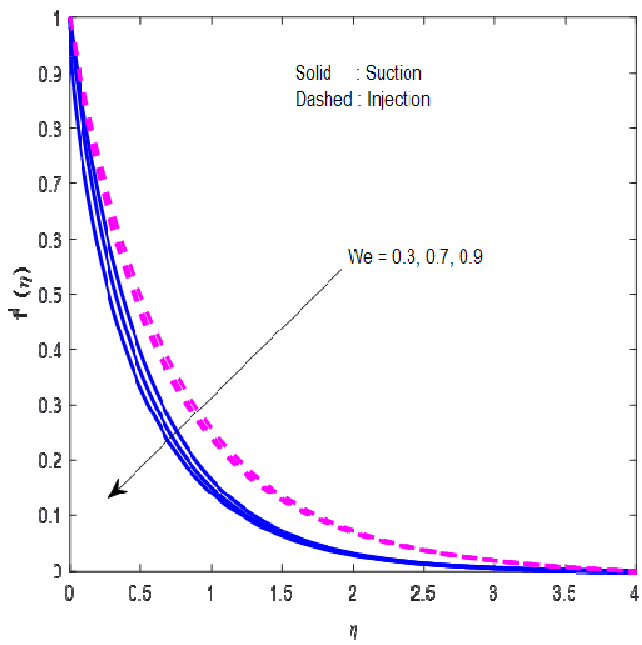

Fig. 5. Consequence of $W e$ on velocity profile

Figures 6-9 explain the impact of $E_{c}, \delta, M$ and $H$ on the temperature profile. Amelioration in viscous dissipation adds additional heat (kinetic energy converts as a heat energy) to the fluid flow. So, temperature rises with the escalation in viscous dissipation (Fig. 6). Figure 7 displays the natural behaviour of thermal buoyancy parameter on fluid temperature (decrement). Due to the rise of Lorentz forces upon the impact of magnetic field parameter, temperature meliorates with bigger $M$ (Fig. 8). Typically, larger heat source parameter causes proliferation of additional heat within the fluid and in turn, assists to enhance the thickness of the thermal boundary layer. So, the heat source parameter escalates fluid temperature (Fig. 9). 

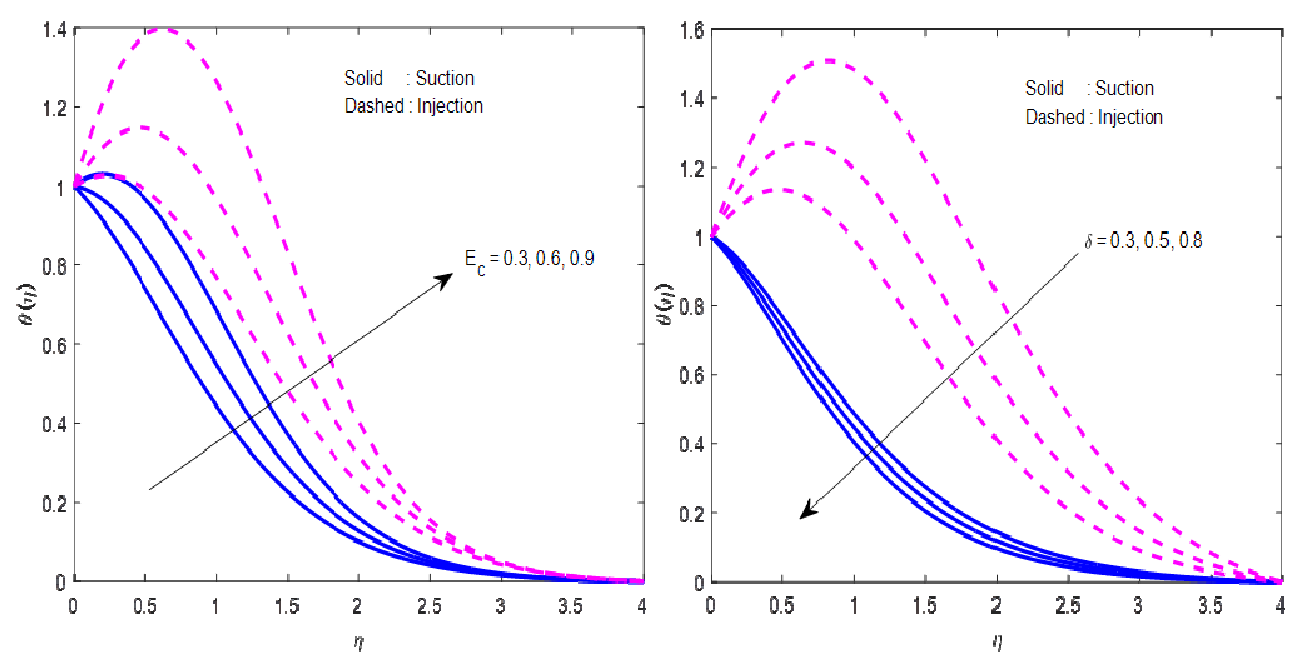

Fig. 6. Consequence of $E_{c}$ on temperature profile

Fig. 7. Consequence of $\delta$ on temperature profile
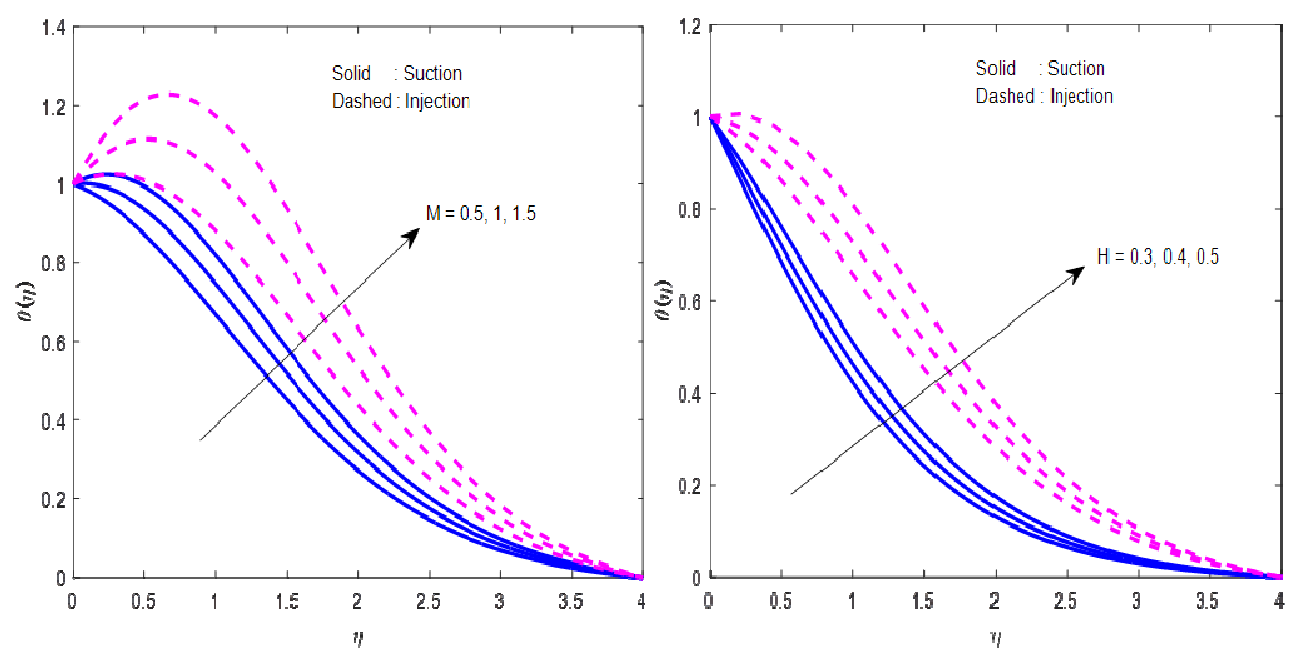

Fig. 8. Consequence of $M$ on temperature profile Fig. 9. Consequence of $H$ on temperature profile

Figures 10 and 11 showed the impact of $F r$ and $W e$ on the skin friction coefficient. It is detected that $\mathrm{Fr}$ lessens the skin friction coefficient and $\mathrm{We}$ meliorates the same. It is evident from Figures 12 and 13 that the Eckert number minimizes the heat transfer rate (The Eckert number ameliorates the thermal boundary layer thickness) and $\delta$ escalates the same ( $\delta$ minimizes the thickness of the thermal boundary layer). Table 1 exhibits the verification of the current results with earlier outcomes for the skin friction coefficient. We found an acceptable agreement. 


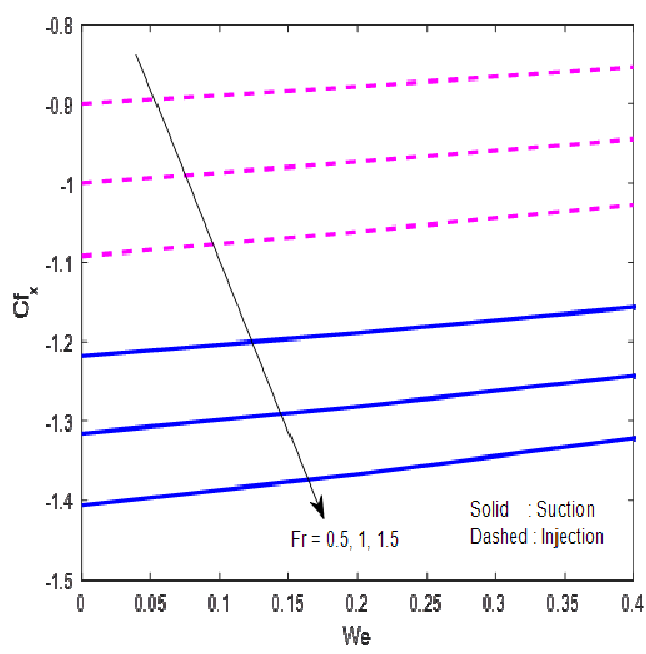

Fig. 10. Consequence of $F r$ on $C f_{x}$

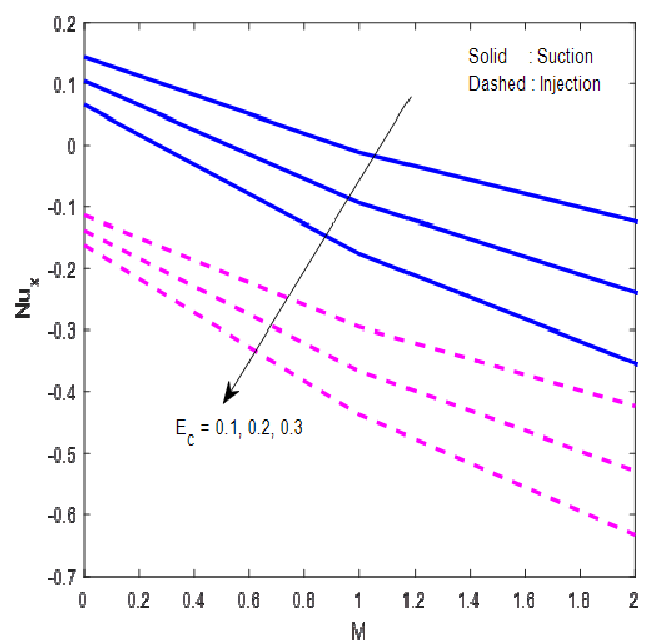

Fig. 12. Consequence of $E_{c}$ on $N u_{x}$

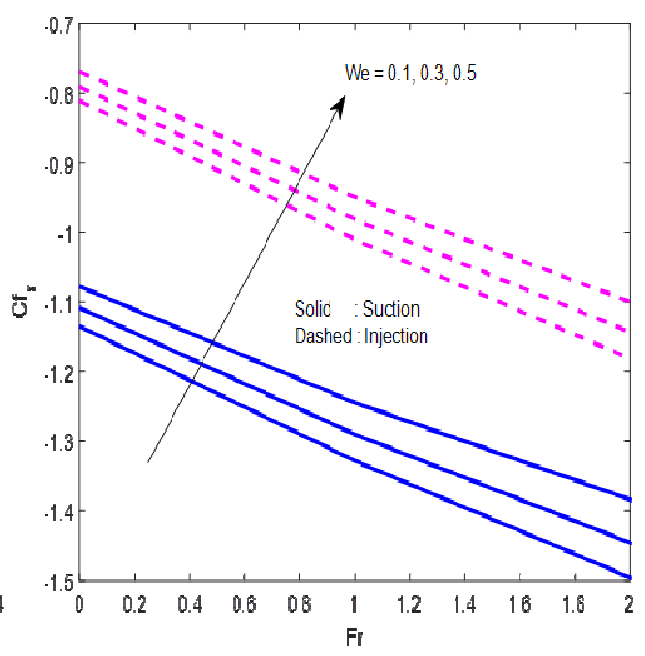

Fig. 11. Consequence of $W e$ on $C f_{x}$

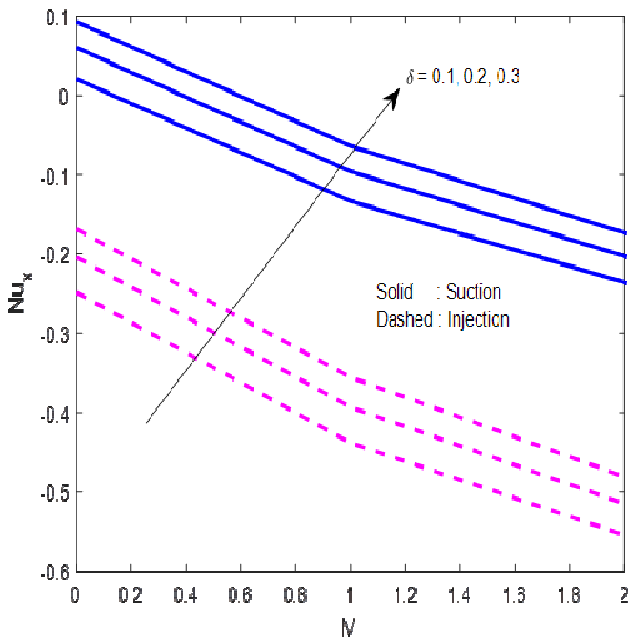

Fig. 13. Consequence of $\delta$ on $N u_{x}$

Table 1. Comparison of the current results with earlier outcomes for skin friction coefficient

\begin{tabular}{|c|c|c|c|c|}
\hline \multirow{2}{*}{$m$} & \multirow{2}{*}{$M$} & \multirow{2}{*}{$W e$} & \multicolumn{2}{|c|}{$C f_{x}$} \\
\cline { 4 - 5 } & & & Akbar et al. [24] & Current result \\
\hline 0 & 0 & 0.3 & 1 & 1 \\
\hline 1 & 0 & 0.3 & 0.94248 & 0.942481 \\
\hline 2 & 0 & 0.3 & 0.88023 & 0.880232 \\
\hline 3 & 0.5 & 0.3 & 0.98804 & 0.988047 \\
\hline 3 & 1 & 0.3 & 1.13454 & 1.134549 \\
\hline 3 & 1.5 & 0.3 & 1.26193 & 1.261933 \\
\hline
\end{tabular}




\section{Conclusions}

Tangent hyperbolic fluid is one of the non-Newtonian fluids in which the constitutive equation is valid for low and high shear rates and used mostly in laboratory experiments and industries. The Darcy-Forchheimer flow model is substantial in the fields where the high flow rate effect is the common phenomenon, for instance, in petroleum engineering. With these things in mind, in this article, we analysed mixed convective dissipative Darcy-Forchheimer flow of tangent hyperbolic fluid by an inclined plate with Joule heating. Primary findings of the current study are summarized as follows:

- The Power lax index parameter lowers the velocity profile.

- Escalation in fluid velocity occurs with the larger thermal buoyancy parameter.

- Temperature rises with the escalation in viscous dissipation.

- The thermal buoyancy parameter minimizes the fluid temperature.

- The Forchheimer number lessens both fluid velocity and skin friction coefficient.

- The heat source parameter escalates fluid temperature, and the Eckert number minimizes the heat transfer rate.

- The Weissenberg number lowers the fluid velocity and meliorates the skin friction coefficient.

\section{References}

[1] Khader, M.M. (2019). Fourth-order predictor-corrector FDM for the effect of viscous dissipation and Joule heating on the Newtonian fluid flow. Computers \& Fluids, 182, 9-14.

[2] Patel, H.R., \& Singh, R. (2019). Thermophoresis, Brownian motion and non-linear thermal radiation effects on mixed convection MHD micropolar fluid flow due to nonlinear stretched sheet in porous medium with viscous dissipation, joule heating and convective boundary condition. International Communications in Heat and Mass Transfer, 107, 68-92.

[3] Mittal, A.S., Patel, H.R., \& Darji, R.R. (2019). Mixed convection micropolar ferrofluid flow with viscous dissipation, joule heating and convective boundary conditions. International Communications in Heat and Mass Transfer, 108, 104320.

[4] Shamshuddin, M., Mishra, S.R., Bég, O.A., \& Kadir, A. (2019). Viscous dissipation and joule heating effects in non-Fourier MHD squeezing flow, heat and mass transfer between Riga plates with thermal radiation: variational parameter method solutions. Arabian Journal for Science and Engineering, 44(9), 8053-8066.

[5] Waqas, M., Dogonchi, A.S., Shehzad, S.A., Khan, M.I., Hayat, T., \& Alsaedi, A. (2020). Nonlinear convection and joule heating impacts in magneto-thixotropic nanofluid stratified flow by convectively heated variable thicked surface. Journal of Molecular Liquids, 300, 111945.

[6] Kumar, A., Tripathi, R., Singh, R., \& Chaurasiya, V.K. (2020). Simultaneous effects of nonlinear thermal radiation and Joule heating on the flow of Williamson nanofluid with entropy generation. Physica A: Statistical Mechanics and its Applications, 551, 123972.

[7] Devi, S., \& Sharma, M.K. (2020). MHD Boundary layer flow over a cone embedded in porous media with Joule heating and viscous dissipation. Defect and Diffusion Forum, 401, 131-139.

[8] Alaidrous, A.A., \& Eid, M.R. (2020). 3-D electromagnetic radiative non-Newtonian nanofluid flow with Joule heating and higher-order reactions in porous materials. Scientific Reports, 10(1), $1-19$. 
[9] Gayatri, M., Reddy, K.J., \& Babu, M.J. (2020). Slip flow of Carreau fluid over a slendering stretching sheet with viscous dissipation and Joule heating. SN Applied Sciences, 2(3), 1-11.

[10] Shoaib, M., Raja, M.A.Z., Sabir, M.T., Awais, M., Islam, S., Shah, Z., \& Kumam, P. (2021). Numerical analysis of 3-D MHD hybrid nanofluid over a rotational disk in presence of thermal radiation with Joule heating and viscous dissipation effects using Lobatto IIIA technique. Alexandria Engineering Journal, 60(4), 3605-3619.

[11] Khan, M.I., Waqas, M., Hayat, T., \& Alsaedi, A. (2017). A comparative study of Casson fluid with homogeneous-heterogeneous reactions. Journal of Colloid and Interface Science, 498, 85-90.

[12] Nayak, M.K., Hakeem, A.A., Ganga, B., Khan, M.I., Waqas, M., \& Makinde, O.D. (2020). Entropy optimized MHD 3D nanomaterial of non-Newtonian fluid: a combined approach to good absorber of solar energy and intensification of heat transport. Computer Methods and Programs in Biomedicine, 186, 105131.

[13] Khan, M.I., \& Alzahrani, F. (2020). Entropy-optimized dissipative flow of Carreau-Yasuda fluid with radiative heat flux and chemical reaction. The European Physical Journal Plus, 135(6), 1-16.

[14] Khan, M.I., Qayyum, S., Kadry, S., Khan, W.A., \& Abbas, S.Z. (2020). Irreversibility analysis and heat transport in squeezing nanoliquid flow of non-Newtonian (second-grade) fluid between infinite plates with activation energy. Arabian Journal for Science and Engineering, 45(6), 4939-4947.

[15] Ijaz Khan, M., \& Alzahrani, F. (2020). Activation energy and binary chemical reaction effect in nonlinear thermal radiative stagnation point flow of Walter-B nanofluid: Numerical computations. International Journal of Modern Physics B, 34(13), 2050132.

[16] Khan, M.I., \& Alzahrani, F. (2020). Binary chemical reaction with activation energy in dissipative flow of non-Newtonian nanomaterial. Journal of Theoretical and Computational Chemistry, $19(03), 2040006$.

[17] Khan, M.I., \& Alzahrani, F. (2020). Transportation of heat through Cattaneo-Christov heat flux model in non-Newtonian fluid subject to internal resistance of particles. Applied Mathematics and Mechanics, 41(8), 1157-1166.

[18] Khan, M.I., \& Alzahrani, F. (2021). Nonlinear dissipative slip flow of Jeffrey nanomaterial towards a curved surface with entropy generation and activation energy. Mathematics and Computers in Simulation, 185, 47-61.

[19] Ibrahim, M., \& Khan, M.I. (2020). Mathematical modeling and analysis of SWCNT-Water and MWCNT-Water flow over a stretchable sheet. Computer methods and programs in biomedicine, $187,105222$.

[20] Ijaz Khan, M., \& Alzahrani, F. (2020). Entropy optimized magnetohydrodynamics Darcy-Forchheimer second order velocity slip flow of nanomaterials between two stretchable disks. Proceedings of the Institution of Mechanical Engineers, Part C: Journal of Mechanical Engineering Science, 234(21), 4190-4199.

[21] Khan, M.I. (2021). Transportation of hybrid nanoparticles in forced convective Darcy-Forchheimer flow by a rotating disk. International Communications in Heat and Mass Transfer, $122,105177$.

[22] Khan, M.I., \& Alzahrani, F. (2021). Free convection and radiation effects in nanofluid (silicon dioxide and molybdenum disulfide) with second order velocity slip, entropy generation, Darcy-Forchheimer porous medium. International Journal of Hydrogen Energy, 46(1), 1362-1369.

[23] Khan, M.I., \& Alzahrani, F. (2021). Dynamics of activation energy and nonlinear mixed convection in Darcy-Forchheimer radiated flow of Carreau nanofluid near stagnation point region. Journal of Thermal Science and Engineering Applications, 13(5), 051009.

[24] Akbar, N.S., Nadeem, S., Haq, R.U., \& Khan, Z.H. (2013). Numerical solutions of magnetohydrodynamic boundary layer flow of tangent hyperbolic fluid towards a stretching sheet. Indian Journal of Physics, 87(11), 1121-1124.

[25] Alam, M.S., Rahman, M.M., \& Sattar, M.A. (2009). On the effectiveness of viscous dissipation and Joule heating on steady magnetohydrodynamic heat and mass transfer flow over an inclined radiate isothermal permeable surface in the presence of thermophoresis. Communications in Nonlinear Science and Numerical Simulation, 14(5), 2132-2143. 